\title{
Caracterización de las prácticas de educación para la sustentabilidad en la modalidad de educación ambiental no formal. Estudio de caso
}

\section{Rosa Herrera Aguilera' ${ }^{1}$, Ana Santa-Anna L. ${ }^{1}$, Alma R. Rivera G. ${ }^{1}$, Elpidio Zárate R. ${ }^{1}$ y Rebeca Patricia Rodríguez Morales ${ }^{2}$}

\author{
${ }^{1}$ Tecnológico Nacional de México. Av. Universidad 1200, Xoco. 03330 Ciudad de \\ México, CDMX, Mexico. \\ ${ }^{2}$ Instituto Tecnológico de Chihuahua - ITChihuahua. Av. Tecnologico 2909, \\ Tecnológico. 31200 Chihuahua, Chih., Mexico.
}

Resumen. La presente investigación tuvo como objetivo principal caracterizar las prácticas de educación para la sustentabilidad en la modalidad de la educación ambiental no formal ejercidas por la Red de Educadores Ambientales de la Ciudad de Chihuahua, México, como un primer ensayo para después caracterizar el perfil de educador ambiental que requiere la problemática ambiental. La metodología es cualitativa con un estudio de caso para estudiar las particularidades de un caso singular para comprender la actividad en circunstancias importantes como lo son las prácticas ambientales que pretenden trascender de la teoría a las acciones concretas y transmisibles requisito de la educación ambiental para la sustentabilidad. La Red de Educadores está conformada por una diversidad de personas profesionistas (edad, ocupación, formación) que comparten una preocupación e interés por mejorar las condiciones ambientales de la ciudad. Las diferentes corrientes de la educación ambiental posibilitan que cada integrante la ejerza a su manera particular; es necesario caracterizar estas prácticas para determinar la pertinencia de estas en la problemática ambiental local e identificar las corrientes adecuadas al contexto. De las 9 prácticas realizadas en el período mayo-junio 2019 se encontraron como predominantes las corrientes conservacionista-resolutiva y en menor proporción la naturalista como categorías tradicionales. Solo una práctica corresponde a la categoría emergente críticasocial. Se consideran las prácticas oportunas a las necesidades del contexto porque fueron propuestas conociendo las necesidades y problemática ambiental de los diferentes estratos sociales de la ciudad. Nos queda claro el reto de formular indicadores de alcances ambientales y perfiles ambientales de los educadores, así como el diseño del modelo pedagógico y objetivos de la Red de Educadores Ambientales.

Palabras claves: Educación ambiental no formal; Categorías educación ambiental; Estudio de caso; Educación ambiental para la sustentabilidad.
Recibido:

$24 / 06 / 2019$

Acepto:

$25 / 08 / 2019$

Disponible en línea: 26/08/2019

Publicado: 31/08/2019

Acceso abierto

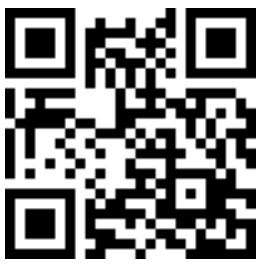

ORCID

(1) 0000-0001-7375-2973 Rosa Herrera Aguilera

(1) 0000-0002-1086-1213 Ana Santa-Anna L.

다 0000-0002-7636-7963 Alma R. Rivera G.

(D) 0000-0002-2523-6955 Elpidio Zárate R.

(D) 0000-0002-6430-7226

Rebeca Patricia Rodríguez Morales 
Abstract. Characterization of education practices for sustainability in the non-formal environmental education modality: Case study. The main objective of the present research was to characterize the practices of education for sustainability in the modality of non-formal Environmental Education exercised by the Environmental Educators Network of the city of Chihuahua, Mexico, as a first essay to later characterize the profile of educator environment that requires environmental problems. The methodology is qualitative with a case study to study the particularities of a singular case to understand the activity in important circumstances such as environmental practices that seek to transcend the theory to concrete and communicable actions requirement of environmental education for sustainability. The Educators Network is made up of a diversity of professional people (age, occupation, training) who share a concern and interest in improving the environmental conditions of the city. The different currents of environmental education allow each member to exercise it in their own particular way; it is necessary to characterize these practices to determine the relevance of these in the local environmental problem and identify the currents appropriate to the context. Of the 9 practices carried out in the May-June 2019 period, conservationistresolutive currents were found to be predominant and, to a lesser extent, the naturalist as traditional categories. Only one practice corresponds to the emergent critical-social category. The opportune practices are considered to the needs of the context because they were proposed knowing the needs and environmental problems of the different social strata of the city. We are clear about the challenge of formulating indicators of environmental scope and environmental profiles of educators, as well as the design of the pedagogical model and objectives of the Network of Environmental Educators.

Keywords: Non-formal Environmental Education; Categories of Environmental Education; Case study; Education for sustainability.

Resumo. Caracterização de práticas educativas para a sustentabilidade na modalidade de Educação Ambiental não formal: estudo de caso. Esta pesquisa teve como objetivo principal caracterizar as práticas de educação para a sustentabilidade na forma de Educação Ambiental não formal exercida pela Rede de Educadores Ambientais na Cidade de Chihuahua, no México, como um primeiro teste para depois caracterizar o perfil de educador ambiental que exige problemas ambientais. A metodologia é qualitativa com um estudo de caso para estudar as particularidades de um caso singular para compreender a atividade em circunstâncias importantes como práticas ambientais que buscam transcender a teoria às exigências concretas e comunicáveis da Educação Ambiental para a sustentabilidade. A Rede de Educadores é composta por uma diversidade de profissionais (idade, ocupação, formação) que 
compartilham uma preocupação e interesse em melhorar as condições ambientais da cidade. As diferentes correntes da Educação Ambiental permitem que cada membro a exerça de maneira particular. É necessário caracterizar estas práticas para determinar a sua relevância no problema ambiental local e identificar as correntes adequadas ao contexto. Das nove práticas realizadas no período de maio a junho de 2019, as correntes conservadoras-resolutivas foram predominantes e, em menor grau, as naturalistas como categorias tradicionais. Apenas uma prática corresponde à categoria crítico-social emergente. As práticas oportunas são consideradas para as necessidades do contexto, pois foram propostas conhecendo as necessidades e os problemas ambientais dos diferentes estratos sociais da cidade. Ficou claro o desafio de formular indicadores de abrangência ambiental e perfis ambientais dos educadores, bem como o desenho do modelo pedagógico e objetivos da Rede de Educadores Ambientais.

Palavras-chave: Educação Ambiental não formal; Categorias de Educação Ambiental; Estudo de caso; Educação para a sustentabilidade.

\section{Introducción}

El siglo XX se caracterizó por el deterioro de los ecosistemas que ocasionó la alarmante crisis ambiental originada por el modelo de crecimiento económico existente; es en 1987 que en la Comisión Mundial para el Medio Ambiente y el Desarrollo (CMMAD), surge el concepto de desarrollo sustentable como un proceso integral que exige a la sociedad compromisos y responsabilidades en la aplicación del modelo económico, político, ambiental y social, así como los patrones de consumo que determinan la calidad de vida. (Educación Ambiental, 2008) El paradigma de la sustentabilidad es conceptualizado como una nueva forma de relación hombre-ambienteproducción en donde la educación desempeña un rol importante en la formación de personas preocupadas por el ambiente y la sociedad, además de contar con la capacidad de participar en la solución de problemas proponiendo alternativas sustentables en la ejecución de los mismos, posibilitando un cambio hacia una actitud positiva frente a la conservación del medio ambiente (Zambrano, 2011).

La Década de la Educación para un Futuro Sostenible (2005-2014) propone que los planes educativos contribuyan a transitar hacia el desarrollo sustentable, por medio de la educación y con cuatro pilares fundamentales: aprender a conocer, aprender a vivir juntos, aprender a hacer $\mathrm{y}$ aprender a ser; de esta forma se integran principios, valores y prácticas del desarrollo sostenible en todas las facetas de la educación. Más antiguo aún es el concepto de educación ambiental que se hizo oficial en 1972 en Estocolmo y cuyo propósito fundamental es la comprensión de las relaciones complejas naturaleza-sociedad, impulsando valores y comportamientos favorables al ambiente, la educación ambiental se convierte en un factor de cambio en el modelo de desarrollo establecido para reorientarlo hacia la sustentabilidad (Educación Ambiental, 2008).

La Educación para la Sostenibilidad se relaciona y comparte objetivos de proyectos educativos como la educación: para todos, para la 
igualdad de género, para la paz, para la tolerancia y la interculturalidad, siendo la educación ambiental su raíz y precursora (Educación Ambiental, 2008).

Para Novo (2009), la Educación Ambiental y la Educación Ambiental para la Sustentabilidad son sinónimos; las décadas de evolución del concepto de educación ambiental; está se constituyo como una educación a favor de un nuevo modelo de desarrollo. Las estrategias y propuestas de la educación ambiental respetan los límites y condiciones del planeta buscando una vida digna para todos los seres humanos convirtiéndose así en un instrumento educativo para el desarrollo sustentable buscando sociedades más justas y equilibradas en el aspecto ambiental y social.

Una vez esclarecido que el hablar de educación ambiental; nos referimos a una educación ambiental para la sustentabilidad, mencionaremos brevemente las bondades de esta y el impacto e importancia para el logro de una sociedad comprometida con el medio ambiente.

La educación es una vía útil y necesaria para potenciar la formación y capacitación ambiental de la sociedad, desde quienes tienen en sus manos la toma de decisiones importantes hasta los niveles ciudadanos, en los que la actuación diaria incide en forma directa sobre el medio (Novo, 1996). Para que la educación alcance su objetivo debe de integrar la dimensión ambiental en los currículos y planes de acción; entendiendo como dimensión ambiental a valores, actitudes y motivaciones que rigen las relaciones sociedad-naturaleza y las formas en que se traducen en los sistemas de producción y apropiación de los recursos productivos; el manejo de los recursos naturales; el equilibrio ecológico y la preservación (Utria, 1986).

La educación ambiental (EA) es un proceso dinámico y participativo que pretende generar una conciencia ambiental que permita identificar la problemática ambiental y emprender acciones de remediación, conservación y prevención del medio ambiente y los recursos naturales $\mathrm{y}$ presenta tres categorías:

a) Formal (EAF): inmersa en el sistema educativo tradicional $y$ organizado en un currículo transversal.

b) No formal (EANF): transmisión de conocimientos, aptitudes y valores ambientales, fuera del sistema educativo para la adquisición de actitudes positivas al medio ambiente. Desde 1967, en la Conferencia Internacional sobre la Crisis Mundial de la Educación, en Virginia, Estados Unidos la UNESCO, plantea un énfasis especial en la necesidad de desarrollar medios educativos diferentes a los convencionalmente escolares.

c) Informal: se da de forma espontánea, no planificada y no responde a estructuras pedagógicas.

El objetivo de esta investigación es el de caracterizar las prácticas realizadas por la Red de Educadores Ambientales en la Ciudad de Chihuahua, Chih., México; desde la Educación Ambiental no formal.

La educación ambiental no formal se desarrolla paralela e independiente de la educación ambiental formal, no se presenta acreditación o certificación y su función es la de reforzar, completar, suplir o continuar contenidos educativos oficiales. En la mayoría de los casos no es impartida por profesionales de la educación y representa una oportunidad de los participantes de asistir voluntariamente. La EANF genera cambios políticos-sociales-económicos y culturales y con el objetivo de desarrollar proyectos que aborden problemas ambientales plenamente identificados y contextualizados rompiendo con las estructuras rígidas de la EAF, se basa en un currículum flexible que se nutre de todas las disciplinas y de los recursos humanos y biológicos disponibles.

En nuestro caso, al igual que el resto del mundo, la EANF se está 
implementando en grupos sociales locales comprometidos con el ambiente la sociedad. La naturaleza inclusiva e interdisciplinaria de la EANF privilegia el aprendizaje, el conocimiento y la puesta en práctica de lo aprendido en un contexto real presenta también desafíos metodológicos que impiden la implementación exitosa de programas de EANF. La UNESCO (2009) formularon el documento titulado Aportes para la Elaboración de Políticas Educativas en el cuál se plantean desafíos para la EANF: a) el establecimiento de objetivos y metas con un diseño pedagógico adecuado al contexto.

b) La identificación de la problemática ambiental local.

c) Disposición de recursos materiales y humanos.

d) La formulación de indicadores que permitan reconocer los procesos y logros educativos que alcanzan los participantes.

e) La construcción de un perfil de educador apropiado.

Tabla 1. Corrientes de la educación ambiental.

\begin{tabular}{|c|c|c|}
\hline Corriente & Concepción del ambiente & $\begin{array}{l}\text { Objetivo de la EA } \\
\end{array}$ \\
\hline Naturalista & Naturaleza & $\begin{array}{c}\text { Reconstruir la relación de pertenencia con la } \\
\text { naturaleza }\end{array}$ \\
\hline Conservacionista & Recurso & Conservación y gestión del medio ambiente \\
\hline Resolutiva & Problema & $\begin{array}{c}\text { Resolución de problemas, diagnóstico de } \\
\text { acción }\end{array}$ \\
\hline Sistémica & Sistema & $\begin{array}{c}\text { Trabajo interdisciplinario que considera } \\
\text { complejidad del sistema }\end{array}$ \\
\hline Científica & Objeto de conocimiento & $\begin{array}{l}\text { Conocimientos y habilidades científicas } \\
\text { transdisciplinarias }\end{array}$ \\
\hline Humanista & Naturaleza-cultura & Abordar patrimonio cultural y natural \\
\hline Moral/ética & Conjunto de valores & Desarrollo de valores ambientales \\
\hline Holística & Totalidad de realidad & $\begin{array}{l}\text { Conocimiento de múltiples dimensiones de } \\
\text { realidades socio-ambientales }\end{array}$ \\
\hline Bio-regionalista & Territorio & Espacio geográfico e identidad comunitaria \\
\hline Práxica & Lugar de convergencia & $\begin{array}{l}\text { Aprendizaje reflexivo en la acción de un } \\
\text { proyecto }\end{array}$ \\
\hline Crítica-social & Objeto de transformación & $\begin{array}{l}\text { Análisis de dinámicas sociales y problemas } \\
\text { ambientales }\end{array}$ \\
\hline Feminista & Relaciones de género & $\begin{array}{l}\text { Reconstruir las relaciones de género en la } \\
\text { participación de proyectos }\end{array}$ \\
\hline Etnográfica & Cultura de referencia & $\begin{array}{l}\text { Carácter cultural de la relación con el medio } \\
\text { ambiente. }\end{array}$ \\
\hline Eco-educación & Esfera de interacción & $\begin{array}{l}\text { aprovechar la relación con el medio } \\
\text { ambiente como crisol de desarrollo personal }\end{array}$ \\
\hline Sustentabilidad & $\begin{array}{l}\text { Economía-sociedad- } \\
\text { naturaleza }\end{array}$ & $\begin{array}{c}\text { Visión integrada de preocupaciones } \\
\text { económicas, sociales y ambientales en una } \\
\text { perspectiva de sostentabilidad. }\end{array}$ \\
\hline
\end{tabular}

Fuente: Adaptado de Sauvé (2004).

La Ciudad de Chihuahua al igual que muchas otras ciudades del mundo enfrente problemas ambientales urgentes como la excesiva generación de residuos sólidos, los escases de agua potable, mala calidad de aire $y$ contaminación de los ecosistemas naturales. Los gobiernos municipales y 
locales han implementado acciones preventivas y correctivas en colaboración con instituciones educativas y sociedad civil. A pesar de los esfuerzos conjuntos los problemas ambientales siguen en aumento y es en esta situación de crisis en donde se integra la Red de Educadores Ambientales por un heterogéneo grupo de personas respecto a edad y formación académica que comparten la preocupación por el medio ambiente y la práctica de la EANF. La red se encuentra en proceso de consolidación, desde el mes de enero del presente año ha venido realizando una serie de prácticas ambientales que se pretenden caracterizar siendo el primer paso para establecer objetivos y modelos pedagógicos adecuados a la problemática ambiental del contexto.

La importancia actual de la educación ambiental ha generado diversas acciones a nivel mundial para realizar las prácticas educativas ambientales desde diferentes enfoques; ante la gran diversidad de teorías y prácticas, Sauvé propone agrupar proposiciones semejantes en categorías considerando divergencias, puntos comunes, oposición y complementariedad formando 15 "corrientes" que no son mutuamente excluyentes; no son categorías rígidas que deforman la realidad, se constituyen como una herramienta de análisis para explorar las diversas proposiciones pedagógicas de la educación ambiental entendiendo como "corriente" a la manera de concebir y practicar la educación ambiental. De manera muy breve la Tabla 1 define las corrientes de educación ambiental (Sauvé, 2004).

\section{Metodología}

El presente trabajo exploratorio y descriptivo en un estudio de caso con enfoque cualitativo que se centra en el análisis, descripción de los fenómenos y cosas observadas. (Combessie et al., 1998) El objetivo es la caracterización de las prácticas realizadas en el período enero-junio 2019. Los datos fueron obtenidos por la interpretación y comprensión del contexto en que se realizan y con la participación activa del investigador en el proceso de investigación. Se considera el estudio de caso el más apropiado para esta investigación porque permite abordar el caso particular de la Red de educadores ya que Candela (2012) describe $a$ "el estudio de caso es el estudio de la particularidad y la complejidad de un caso singular, para poder llegar a comprender su actividad en circunstancias importantes".

Se considera exploratorio porque no existe ningún antecedente de la caracterización de prácticas de educación ambiental en la Red de Educadores Ambientales.

El enfoque descriptivo permite contrastar los datos obtenidos sobre las variables y factores que guardan relación con el problema de investigación usando datos cualitativos, que son obtenidos a partir de entrevistas a los dirigentes de las prácticas educativas ambientales.

La metodología de estudio de caso está basada en el paradigma cognitivo-constructivista, el proceso de investigación es una observación participativa en donde el investigador y los sujetos-actores comparten un contexto de trabajo (Candela, 2012).

Se realizaron entrevistas estructuradas a 9 integrantes de la red quienes tuvieron a cargo el diseño y realización de las 9 prácticas de Educación ambiental no formal. El formato de entrevista indaga sobre las características socio demográficas de los líderes y del, para conocer elementos personales que incidan en su desempeño profesional $y$ porque el nivel de educación al igual que la experiencia inciden en el diseño y realización de la práctica. La indagación sobre el enfoque o modelo de educación ambiental se realiza debido a que estos modelos han evolucionado y se pretende saber si están de acuerdo al contexto actual o en 
cual se ubican. Para esto se establecieron 4 opciones conforme a las definiciones que se presentaron en el marco teórico de este documento y que son propuestas por (Sauvé, 2004).

Para indagar sobre las prácticas de la EANF se procedió a realizar una caracterización de la Red de educadores ambientales y a la aplicación entrevistas a responsables de las prácticas propuestas; para tal fin existió un acercamiento previo con cada una de las 9 personas para explicarles los alcances de la investigación.

\section{Resultados}

La Red de Educadores Ambientales (REACH) es una asociación sin fines de lucro en proceso de registro de asociación civil: fue conformada en los años 1990 estando en función por algunos años, permaneció inactiva hasta enero del presente año y por gestiones de un grupo de profesionistas preocupados por el deterioro ambiental de la ciudad proponen conformar y activar la Red. La totalidad de los 15 integrantes activos de la red son profesionistas que residen en la ciudad y con edades entre los 23-70 años; la mayoría tiene formación en Ecología, seguido por docencia, Química $\mathrm{y}$ Agronomía. El 40\% de los integrantes posee estudios de posgrado a nivel maestría y doctorado; otro $40 \%$ se encuentra cursando estudios de maestría. La red se reúne mensualmente con el objetivo principal de difundir y practicar la EANF en esta ciudad $y$ municipios cercanos.

En conmemoración del día mundial del medio ambiente y para ejercer acciones ambientales que requiere la EANF se diseñaron $y$ realizaron 9 actividades dirigidas por un líder integrante de la Red en los meses mayo-junio 2019, los recursos económicos fueron autofinanciados y se contó con apoyo en infraestructura y transporte por parte de instituciones educativas.

Los responsables de las prácticas realizadas por la Red de educadores, en su mayoría sobrepasan los 50 años de edad, con amplia experiencia en el campo de la EANF y del género masculino.

Tabla 2. Características socio demográficas y educativas de los responsables de las actividades ambientales.

\begin{tabular}{|c|c|l|c|c|c|}
\hline \multicolumn{2}{|c|}{ Edad } & Género & Formación & $\begin{array}{c}\text { Años experiencia } \\
\text { EANF }\end{array}$ & Ocupación \\
\hline 1 & 24 & Femenino & Biología & 1 & Estudiante posgrado \\
\hline 2 & 58 & Femenino & Química & 30 & Docente \\
\hline 3 & 27 & Masculino & Ecología & 2 & Sector ambiental \\
\hline 4 & 59 & Masculino & Agronomía & 30 & Sector ambiental \\
\hline 5 & 58 & Femenino & Maestra & 30 & Jubilación \\
\hline 6 & 30 & Femenino & Ecología & 3 & Sector ambiental \\
\hline 7 & 63 & Masculino & Veterinario & 30 & Sector ambiental \\
\hline 8 & 23 & Masculino & Ecología & 2 & Sector ambiental \\
\hline 9 & 65 & Masculino & Zootecnia & 30 & \\
\hline
\end{tabular}


Tabla 3. Caracterización de prácticas ambientales.

\begin{tabular}{|l|l|l|l|}
\hline & Práctica & Objetivo & Corriente EA \\
\hline 1 & Juego de la basura & $\begin{array}{l}\text { Fomentar la importancia y necesidad } \\
\text { de separar basura. Actividad lúdica } \\
\text { diseñada para niños de primaria. }\end{array}$ & $\begin{array}{l}\text { Conservacionista } \\
\text { Resolutiva }\end{array}$ \\
\hline 2 & Taller plástico-pet-problema & $\begin{array}{l}\text { Identificación de diferentes materiales } \\
\text { plásticos y fomentar la separación, } \\
\text { reuso y reciclado. Taller diseñado para } \\
\text { personal y maestros de educación } \\
\text { especial. }\end{array}$ & $\begin{array}{l}\text { Conservacionista } \\
\text { Resolutiva }\end{array}$ \\
\hline 3 & Taller carta de la tierra & $\begin{array}{l}\text { Difusión de la carta en jóvenes para } \\
\text { posterior replicación del taller en } \\
\text { niños y adolescentes. }\end{array}$ & Conservacionista \\
\hline 4 & Taller huertos sustentables & $\begin{array}{l}\text { Aprovechar y conservar las especies } \\
\text { endémicas de la localidad. Taller } \\
\text { diseñado para fraccionamientos } \\
\text { periféricos de la ciudad. }\end{array}$ & $\begin{array}{l}\text { Naturalista } \\
\text { Conservacionista }\end{array}$ \\
\hline 5 & Reforestación parques & $\begin{array}{l}\text { Restaurar y conservar un espacio } \\
\text { público. }\end{array}$ & $\begin{array}{l}\text { Naturalista } \\
\text { Conservacionista }\end{array}$ \\
\hline 6 & Taller de polinizadores & $\begin{array}{l}\text { Realizar acciones de conservación de } \\
\text { las especies polinizadoras en la ciudad. }\end{array}$ & $\begin{array}{l}\text { Naturalista } \\
\text { Conservacionista }\end{array}$ \\
\hline 7 & Taller para capacitadores & $\begin{array}{l}\text { Formación teórico-práctica para } \\
\text { jóvenes que desempeñan el rol de } \\
\text { capacitadores ambientales. }\end{array}$ & $\begin{array}{l}\text { Conservacionista } \\
\text { Resolutiva }\end{array}$ \\
\hline 9 & Recarga de acuíferos & $\begin{array}{l}\text { Dialogo e intercambio de saberes en } \\
\text { jóvenes que luchan por una justicia } \\
\text { ambiental en la localidad. }\end{array}$ & Crítica-social \\
\hline $\begin{array}{l}\text { Conferencia informativa y fomento a } \\
\text { realizar acciones resolutivas. }\end{array}$ & $\begin{array}{l}\text { Conservacionista } \\
\text { Resolutiva }\end{array}$ \\
\hline
\end{tabular}

\section{Conclusiones}

Las 4 corrientes de la educación ambiental en las que se clasificaron las 9 prácticas realizadas; 3 de ellas corresponden a las categorías tradicionales: naturalista, conservacionista $\mathrm{y}$ resolutiva; solamente la categoría críticasocial se ubica en las corrientes emergentes. Se resalta la importancia de la diversidad de edades y profesiones de los integrantes de la red; ya que la práctica emergente fue realizada por el integrante más joven de la red.

La categoría conservacionista prevalece en 7 de las 9 prácticas realizadas por responsables jóvenes $\mathrm{y}$ adultos (59-65 años) y se enfocan en la conservación de la calidad y cantidad de los recursos naturales; la naturaleza es considerada un recurso y resalta la preocupación por la gestión ambiental y los programas educativos basados en las $3 R^{\prime}$ 's (reduce, recicla y reuso) y engloba acciones de comportamiento individual, proyectos comunitarios y educación para el consumo. En el contexto actual, esta corriente se ve favorecida por una creciente preocupación ambiental y la difusión en medios masivos de comunicación; la oferta de productos ecológicos y biodegradables; el auge de movimientos sociales ambientalistas y las tendencias políticas conservacionistas. El principal objetivo educativo de esta corriente es la de sensibilizar a las personas sobre la problemática ambiental causada por el hombre. Se debe tener cuidado en que los educadores ambientales no presenten una orientación didáctica "catastrofista", presidida por la idea de que el desarrollo científico y tecnológico es perjudicial para el mundo. (Álvarez et al., 2004) 
La corriente resolutiva unida con frecuencia a la conservacionista, tiene como objetivo informar sobre problemáticas ambientales y desarrollar habilidades para resolverlos con la modificación de comportamientos o el desarrollo de proyectos colectivos. El modelo pedagógico se centra en la resolución de problemas ambientales.

En esta ocasión la información teórica se acompañó de acciones ambientales viables y sencillas que permitan reducir el daño al medio ambiente y fueron dirigidas a niños, jóvenes y adultos abordando problemas ambientales del contexto como los son la excesiva generación de basura, espacios públicos deteriorados ambientalmente, conservación de flora y mantos acuíferos.

La corriente naturalista centrada en la relación con la naturaleza en donde esta es considerada como educadora y como un medio de aprendizaje en donde se reconoce el valor intrínseco de la naturaleza. El enfoque educativo puede ser cognitivo, experiencial, afectivo, espiritual o artístico (Sauvé, 2004).

La orientación naturalista identifica al ambiente como un medio físico-biológico y la educación ambiental puede limitarse al conocimiento de ecosistemas locales; la estrategia más sencilla consiste en introducir una nueva materia. Si por el contrario, se parte de una concepción del medio ambiente y de la problemática ambiental que abarque los aspectos físico- naturales, sociales y los político-económicos, la alternativa más indicada es infundir a las disciplinas curriculares un sesgo ambientalista (Flor, 2005). Además existe el riesgo de adoptar una educación ambiental reproductiva que consiste en repetir y acoger lo que instituciones internacionales como la UNESCO determinan como educación ambiental ignorando propuestas locales (Eschenhagen, 2003)

En nuestro caso las prácticas naturalistas fueron dirigidas a grupos de adultos con diferente situación socioeconómica y educativa, atendiendo las necesidades e intereses propios del grupo de personas.

La corriente crítico-social analiza las dinámicas sociales de la problemática ambiental transformando la realidad generando proyectos de acción. Esta corriente se basa en modelos pedagógicos interdisciplinarios para desarrollar un saber-acción para resolver problemas ambientales locales. Insiste en la contextualización de los temas tratados y en la importancia del diálogo de los saberes. (Sauvé, 2004).

La práctica ambiental críticosocial dirigida por jóvenes y para jóvenes conto con participación voluntaria de jóvenes pertenecientes a diversas asociaciones de corte ambiental, a pesar de ser un número reducido de participantes, las aportaciones, propuestas y el dialogo fue activo $y$ enriquecedor lo que demuestra el compromiso y la conciencia ambiental de los jóvenes participantes.

En general podemos concluir que las prácticas realizadas abordaron realidades ambientales de acuerdo al contexto local; la experiencia y formación de los dirigentes de las prácticas fueron plasmadas en las propuestas. La diversidad de formaciones y edades de los integrantes de la Red constituyen una fortaleza, más que una debilidad ya que se cuenta con un interés común en el que cada uno trabaja en su ámbito profesional y personal. Situación que permitió el conocimiento de la problemática ambiental de la ciudad y la disposición de recursos humanos y materiales Este primer ejercicio servirá de base para la planificación de actividades futuras considerando las oportunidades de mejorar y actualizar los trabajos ambientales quedando como tareas pendientes el establecimiento de metas y objetivos con un diseño pedagógico, la formulación de indicadores de logros educativos y la construcción de un perfil adecuado de educador ambiental. Nos queda la certeza del compromiso y capacidades profesionales de los integrantes de la Red 
para contribuir en la medida de nuestras posibilidades a la construcción de un presente y futuro sustentable.

\section{Conflicto de intereses}

Los autores declaran no tener ningún conflicto de intereses.

\section{Referencias}

Álvarez, P; García, J; Fernández, M. Ideología ambiental del profesorado de educación secundaria obligatoria. Implicaciones didácticas y evidencias sobre la validez de un instrumento. Revista Electrónica de Enseñanza de las Ciencias, v. 3, n. 3, p. 385-396, 2004. Disponible en: <http://reec.uvigo.es/volumenes/volumen3 /REEC_3_3_8.pdf $>$. Consultado: 10 jun. 2019.

Candela, B. F. La captura, la documentación y la representación del CPC de un profesor experimentado y "ejemplar" acerca del núcleo conceptual de la discontinuidad de la materia. Valle, Santiago de Cali: Universidad del Valle, Instituto de Educación y Pedagogía, 2012. (Maestría en educación).

Combessie, J.; Cerda, H.; Henao, M.; Sánchez, S.; Bravo, N.; Alvarado, S. Investigación educativa e innovación. Bogotá, Colombia: Cooperativa Editorial Santa Fé, 1998.

Educación Ambiental: principio del desarrollo sustentable. Innovación Educativa, v. 8, n. 43, p. 77-84, 2008. Disponible en: <http://www.redalyc.org/ articulo.oa?id=179421221005>. Consultado: 12 jun. 2019.

Eschenhagen, M. L. El estado del arte de la Educación Ambiental y problemas a los que se está enfrentando. Anales del II Encuentro Metropolitano de Educación Ambiental, Toluca, 2003.
Flor, J. I. Claves de la Educación Ambiental. Santander: Centro de Estudios Montañeses, 2005. Disponible en: <http://centrode estudiosmontaneses.com/wp-content/ uploads/DOC_CEM/BIBLIOTECA/EDICION_O TROS/claves_para_la_educacion_ambiental_2 005.pdf>. Consultado: 10 jun. 2019.

Novo, M. La Educación Ambiental formal y no formal: dos sistemas complementarios. Revista Iberoamericana de Educación, v. 11, p. 75-102, 1996. https://doi.org/ 10.35362/rie1101158

Novo, M. La Educación Ambiental: una genuina educación para el desarrollo sostenible. Revista de Educación, n. extra, p.195-217, 2009. Disponible en: <http://www.revistaeducacion.mec.es/re20 09/re2009_09.pdf>. Consultado: 10 jun. 2019.

UNESCO - Organización de las Naciones Unidas para la Educación, la Ciencia y la Cultura. Aportes para la elaboración de propuestas de políticas educativas. Montevideo, Uruguay: UNESCO, Ministerio de Educación y Cultura de Uruguay, 2009.

Sauvé, L. Una cartografía de corrientes en la Educación Ambiental. Porto Alegre: Artmed, 2004. (Texto académico, 1).

Utria, R. D. La dimensión ambiental del desarrollo y su planificación. Bogotá, Fundación Universidad de Bogotá “Jorge Tadeo Lozano", 1986. Disponible en: <https://repositorio.cepal.org/bitstream/ha ndle/11362/28257/S30131 U92_es.pdf>.

Consultado: 10 jun. 2019.

Zambrano, J. El desarrollo sustentable y los programas educativos ambientales. CONHISREMI, Revista Universitaria Arbitrada de Investigación y Diálogo Académico, v. 7, n. 1, p.68-85, 2011. Disponible en: <https://core.ac.uk/ download/pdf/25868037.pdf>. Consultado: 10 jun. 2019. 